\title{
The vaso-occlusive crisis of sickle cell disease
}

\section{Time for coordinated trials of new treatments}

Sickle cell anaemia was the first molecular disease to be described $^{\prime}$; despite intensive study we still do not understand the relations between the clinical picture, the cellular defects, and the molecular make up of any one person. Two recent papers have further elucidated the complex pathophysiology associated with painful crisis, although their implications for treatment are unclear.

Clinicians have classically considered the basic defect in sickle cell disease to be the polymerisation of the deoxyhaemoglobin $\mathrm{S}$ with consequent membrane damage. Hebble has proposed that we should also take into account the instability of the haemoglobin $\mathrm{S}$ molecule with auto-oxidation and consequent in vivo oxidant damage to the cell membrane. ${ }^{2}$ This results in alteration in the lipid bilayer dynamics, its uncoupling from the membrane skeleton, and increased cation permeability of the membrane. He suggests that the true impact of these membrane defects on the clinical picture is to add a stochastic influence, modulating the fundamental predisposition to disease severity conferred by genetic determinants of haemoglobin polymerisation. He has shown that the adherence of sickle erythrocytes to human endothelial cells in vitro correlates directly with the clinical severity of vaso-occlusive morbidity in individual patients. His hypothesis integrates the decreased stability of haemoglobin $\mathrm{S}$ with the decreased solubility; these both lead to cellular dehydration which, together with the haemoglobin-membrane interaction, results in abnormal microrheology and endothelial adhesivity and also macrophage interaction, phospholipid destabilisation, and protein defects. This work is now being taken further by other workers, including Weinstein et al, who have shown that sickle erythrocytes inhibit the synthesis of DNA in human endothelial cells when applied to endothelial monolayers. ${ }^{3}$ The clinical importance of this abnormal response is unclear, but we know that in the normal endothelial response to injury synthesis of DNA is an early step.

The second paper is by Serjeant and Chalmers, who have proposed a "steal" syndrome as a new explanation of the painful crisis. ${ }^{+}$They suggest that skin cooling is the primary insult, which induces a centrally mediated reflex causing shunting away from the bone marrow with resulting avascular necrosis. Their suggestion is based on limited evidence that the vascular reflexes behave differently in sickle cell disease and on observations made during a questionnaire study of Jamaican patients: many patients volunteered that the pain affects symmetrical joints either simultaneously or within six hours. More interesting than the hypothesis itself is the fact that new ideas are being entertained that bring together different clinical sciences.

These new approaches offer hope to patients with sickle cell disease, about 5000 of them in Britain, but at present the management of the painful crisis remains unchanged. The best way of preventing painful crises is for patients to be aware of both general and personal factors that precipitate them. In particular they should maintain a steady temperature and avoid dehydration at all costs. Any infection should be treated promptly. Psychological stress has been incriminated as a precipitating factor in up to $22 \%$ of episodes of painful crisis, and patients may need counselling support, distraction therapy, and even self hypnosis.

When patients experience pain they should increase their fluid intake, take oral analgesia such as paracetamol, rest, and keep warm. If the pain does not respond, they have an associated constitutional upset, they develop other symptoms or signs, or they know from personal experience that this crisis will develop into a severe one, they should present to the nearest casualty department. Patients who know they have sickle cell disease should be believed and given immediate parenteral analgesia, usually an opiate. There is now a move away from pethidine as the large doses required may induce fits through an accumulation of nor-pethidinic acid. It is reasonable to take a clinical history and, once the pain is relieved, to perform a physical examination. Particular care should be taken to exclude signs of complications. These include the sickle chest syndrome, the "girdle syndrome," neurological deficit, and priapism. ${ }^{5}$ Fluids are generally given intravenously as clear fluids. When the pain is confined to the limbs the average period of admission is five days; this rises to 10 days if the pain is more centrally distributed in the trunk. ${ }^{6}$

Transfusion of red cells is rarely necessary for a straightforward severe painful crisis. Indeed, the problems associated with repeated blood transfusion, including the risks of alloimmunisation, ${ }^{7}$ transmission of infection, and the accumulation of iron, have led to increasingly strict criteria for transfusion in sickle cell disease. ${ }^{8}$ Transfusion is indicated in the severe chest syndrome (when arterial oxygen tension is less than $8 \mathrm{kPa}$ with the patient breathing air) and for acute neurological deficit. If the patient's haemoglobin concentration is normal for that patient and transfusion is required then exchange transfusion should be used. ${ }^{8}$

Two chemotherapeutic agents, azacitidine (5-azacytidine) and hydroxyurea, have been shown to raise fetal haemoglobin production and should, as a result, ameliorate the clinical 
picture. Azacitidine has been dropped from clinical use, but a preliminary report of treatment with hydroxyurea is promising. In three patients there was a $2 \cdot 3$-fold to 16 -fold increase in the percentage of haemoglobin $F$ and a virtual cessation of painful crises. ${ }^{9}$ The results of the careful controlled study of hydroxyurea being undertaken for the National Institutes of Health are awaited with interest, although the way hydroxyurea raises the haemoglobin $\mathrm{F}$ concentration is still incompletely understood.

Bone marrow transplantation for sickle cell disease has now been performed in a few centres, mainly in Belgium. ${ }^{10}$ Though transplantation is curative, it raises ethical issues that have yet to be fully discussed by either health professionals or patients in Britain. We need to explore these, as for $\beta$ thalassaemia major.

There is an urgent need in Britain to develop a structure for developing coordinated trials of new treatments for sickle cell disease. As a model haematologists have the excellently run trials for the leukaemias sponsored by the Medical Research Council. These not only produced valuable scientific infor- mation but also enabled physicians with only a few patients under their care to use up to date treatments.

Consultant Haematologist,

Central Middlesex Hospital,

London NW10 7NS

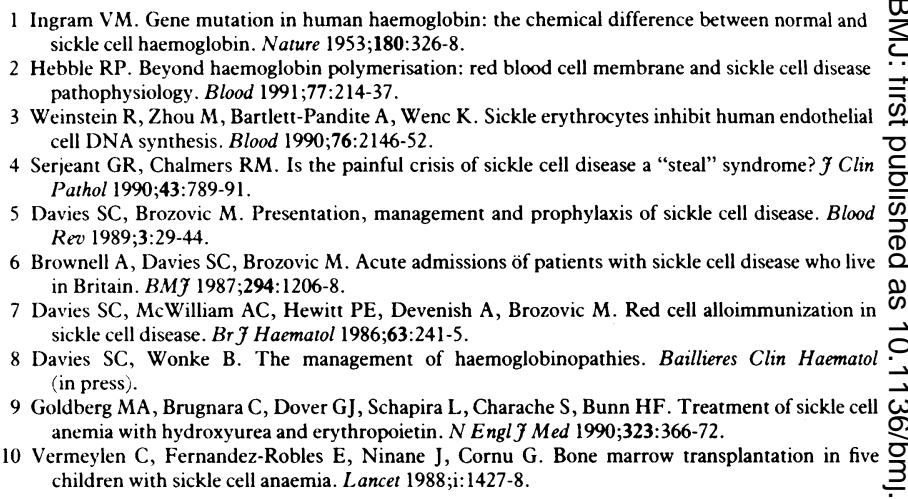

\section{Hepatitis A}

\section{Changing prevalence and possible vaccines}

Although hepatitis A is generally a benign infection, it may have a protracted or relapsing course, ${ }^{12}$ and, very rarely, result in fulminant hepatic failure. Its severity increases with age; in England and Wales in 1980-8 the fatality rate was 0.02 $0.03 \%$ in those aged under 55 but $1.5 \%$ in those aged over $64 .^{3}$

Hepatitis A virus is exceptionally stable and is spread by the faecal-oral route, either from person to person or through contaminated food or water. In countries with poor sanitation this is common-with almost universal infection of young children resulting in little clinical disease and lifelong immunity. As standards of hygiene improve, however, infection is delayed, and many escape early asymptomatic infection only to develop clinical hepatitis A as adolescents or adults. This emergence of the disease at an older age is one of several factors giving a sharper edge to the search for a vaccine.

In many developed countries the prevalence of immunity is related to age and is falling. Among medical students in London the prevalence of hepatitis A IgG fell from $14 \%$ in $1980^{4}$ to $6 \%$ in 1990 (unpublished findings), and in blood donors in London it fell from $47 \%$ in 1977 to $32 \%$ in 1985.5 Among 863 Foreign Office staff the overall prevalence of hepatitis A IgG in $1989-90$ was $39 \%$, being $43 \%$ in those over 40 but only $14 \%$ in younger people (unpublished findings).

The incidence of hepatitis $\mathrm{A}$ is difficult if not impossible to measure, as many cases are asymptomatic and there is considerable underreporting. Despite the decreasing seroprevalence, notifications and laboratory reports of hepatitis A to the Public Health Laboratory Service Communicable Disease Surveillance Centre increased between 1987 and 1990. Although this may reflect increased reporting, it is more likely that infection is spreading in the community. Indeed, hepatitis A virus infection is now endemic in several deprived urban areas including Liverpool - where it has been endemic in children for over a year ( $Q$ Syed, personal communication). If this trend continues hepatitis A may become a marker of inner city deprivation.

Hepatitis A has traditionally been controlled by sewage disposal and better hygiene, while human normal immuno- $\vec{\emptyset}$ globulin has been used in developed countries as prophylaxis. both before and after exposure. In the United Kingdom use of ${ }_{0}$ this preparation has increased recently, mostly because of travel to endemic countries. About 1.5 million people visit $\overline{0}$ such areas annually, about 600000 for the first time ( $M \stackrel{0}{\circ}$ Capaldi, personal communication); in 1990 the number of doses of human normal immunoglobulin issued approached $\overrightarrow{\overrightarrow{0}}$ 600000 . This plasma product is safe and effective, but it may become less effective with the declining prevalence of immunity to hepatitis A. Furthermore, it is costly and gives only shortlived protection.

A vaccine would have obvious advantages. Several types are possible, but conventional inactivated vaccines and live 3 attenuated vaccines have received most attention. Initial studies in primates with live attenuated vaccines identified several variants that were safe, immunogenic, and protec-o tive.$^{67}$ In human volunteers some difficulty was experienced in achieving a balance between immunogenicity and attenuation, ${ }^{8-10}$ but encouraging results have been reported by $a$ 을 Chinese group using a different attenuated strain, which induced high seroconversion rates without serious side N effects. ${ }^{1112}$ Furthermore, this vaccine provided protection ${ }_{\sigma}^{\omega}$ under natural conditions. In all these studies live vaccine has been given by injection; the oral route would be preferable but $\frac{}{\mathbb{D}}$ so far results have been disappointing. Points of concern $\stackrel{?}{+}$ include excretion and transmission of the virus, reversion to 0 virulence, and the possibility of persistence in the liver or $\frac{\vec{\Phi}}{\vec{D}}$ elsewhere. Moreover, a chemical or genetic marker for $\frac{\widehat{\Phi}}{\Phi}$ attenuation remains to be identified.

Studies with inactivated vaccines in large numbers of human volunteers have shown that these vaccines are safe, 20 induce high seroconversion rates with just two doses givens one month apart, and induce neutralising antibodies at $\frac{\bar{\partial}}{\partial}$ concentrations that usually exceed the peak concentration. after one dose of human normal immunoglobulin. ${ }^{13-15} \mathrm{~A}$ third dose results in higher concentrations of antibody, which should provide long term protection. As with hepatitis B 